\title{
El midodrine disminuye la recurrencia de síncope vaso vagal en pacientes jóvenes
}

Midodrine decreases the recurrence of vasovagal syncope in young patients

\section{Comentado de:}

Sheldon R, et al. Ann Intern Med. 2021. doi: 10.7326/M205415. Epub ahead of print. PMID: $34339231^{1}$

\section{Objetivo}

Determinar si el midodrine puede prevenir el síncope vasovagal en condiciones clínicas habituales.

\section{Diseño, lugar y población}

Ensayo clínico aleatorizado, controlado y doble ciego realizado en 25 hospitales universitarios de Canadá, Estados Unidos, México y el Reino Unido.

Fueron incluidos pacientes adultos con diagnóstico de síncope vasovagal recurrente sin condiciones comórbidas graves.

\section{Intervención}

Los pacientes fueron asignados aleatoriamente (1:1) al grupo placebo $o$ al grupo midodrine.

La dosis de midodrine fue de $5 \mathrm{mg}$, administrada 3 veces por día por vía oral, pudiendo aumentarse hasta $10 \mathrm{mg}$ por dosis según la tolerancia del paciente.

\section{Medición de resultados principales}

En ambos grupos fue registrada la proporción de pacientes que sufrieron al menos un episodio de síncope durante doce meses de seguimiento.

El estadístico de log-rank fue utilizado para comparar las curvas de incidencia de síncope, y los cocientes de riesgo (hazard ratio [HR]) estimados mediante modelos de regresión de Cox, para comparar el tiempo al primer evento en ambas ramas de tratamiento.

\section{Resultados principales}

Fueron incluidos 133 pacientes adultos, sanos desde el punto de vista cardiovascular, con una mediana de 32 años de edad y una mediana de 6 (rango intercuartilo 3 a 20) de episodios sincopales en el último año; el $73 \%$ fueron mujeres.

Como se presenta en la Tabla 1, en comparación con el grupo que recibió placebo, en el grupo midodrine se observó una menor proporción de pacientes con al menos un episodio de síncope.

El tiempo hasta la recurrencia del evento fue significativamente mayor en el grupo midodrine (HR 0,59; intervalo de confianza [IC] del 95 , 0,37 a 0,96); $p=0,035$; log-rank $p=0,031$ ). Los efectos adversos que presentaron los pacientes fueron parestesia, piloerección, cefaleas y náuseas, sin diferencias significativas entre los grupos. Dos pacientes del grupo midodrine abandonaron el estudio por hipertensión y dos del grupo placebo, por náuseas y cefaleas.

\section{Conclusiones}

El uso de midodrine puede reducir la recurrencia de síncope en pacientes jóvenes y sanos, con síncopes vasovagales frecuentes.

Fuente de financiamiento / Conflictos de interés de los autores: El estudio fue financiado con el subsidio MOP 115124 del Instituto de Investigación en Salud Canadiense, Ottawa, Canadá, y por donaciones de placebo de Shire Pharmaceuticals y Apotex. Dos autores declararon haber recibido honorarios y subsidios no relacionados con este trabajo, los demás autores no informaron conflictos de interés.

Tabla 1. Proporción de pacientes que presentaron al menos un episodio sincopal al año de seguimiento en cada rama del estudio y medidas de efecto estimadas

\begin{tabular}{|l|c|c|c|c|c|}
\hline Desenlace & $\begin{array}{c}\text { Grupo midodrine, } \\
\mathrm{n} / \mathrm{N}(\%)\end{array}$ & $\begin{array}{c}\text { Grupo placebo, } \\
\mathrm{n} / \mathrm{N}(\%)\end{array}$ & $\begin{array}{c}\text { Riesgo Relativo } \\
(\mathrm{IC} 95 \%)\end{array}$ & $\begin{array}{c}\text { Reducción de riesgo } \\
(\mathrm{IC} 95 \%)\end{array}$ & $\begin{array}{c}\text { Número Necesario a } \\
\text { Tratar (IC 95\%) }\end{array}$ \\
\hline $\begin{array}{l}\text { Pacientes con al menos un } \\
\text { episodio sincopal }\end{array}$ & $28 / 66(42)$ & $41 / 67(61)$ & $\begin{array}{c}0,69 \\
(0,49 \text { a 0,97) }\end{array}$ & $\begin{array}{c}0,19 \\
(0,02 \text { a } 0,36)\end{array}$ & $\begin{array}{c}5,3 \\
(2,8 \text { a 47,6) }\end{array}$ \\
\hline
\end{tabular}

\section{Comentario}

El síncope vasovagal es un problema frecuente, que puede producir daño en quienes lo experimentan, por traumatismos secundarios a las caídas, y que además deteriora la calidad de vida ${ }^{2,3}$. Sin embargo, previo al estudio comentado, se carecía de información de alta calidad sobre qué fármacos podrían ser utilizados para prevenirlos.

Dado que esta patología surge muchas veces cuando fisiopatológicamente se combina una disminución del volumen eyectivo con una vasodilatación periférica y un aumento de la complacencia venosa, era de esperar que el midodrine tenga un rol beneficioso, al ser un agonista alfa-1 directo que produce vasoconstricción arterial y venosa ${ }^{4}$.

Los hallazgos de este estudio muestran que el midodrine es efectivo para reducir la recurrencia de síncope. Podría utilizarse de manera conjunta con las medidas no farmacológicas básicas, como los cambios en el régimen alimentario, aumento del consumo de sal y de la ingesta de líquidos ${ }^{5}$, compresión periférica y/o plan de ejercicios reglado ${ }^{6}$.

Dentro de las limitaciones del estudio se destacan el pequeño tamaño muestral, un período de observación relativamente corto, que sólo se incluyeron pacientes jóvenes y sanos, y que una alta proporción fue reclutada en uno de los centros. 
En línea con las conclusiones del estudio, cabe destacar que el midodrine puede ser útil no sólo en el síncope vasovagal, sino en otras patologías asociadas que cursan con hipotensión ortostática.

\section{Conclusiones de los comentadores}

Los hallazgos de este estudio internacional demuestran que el midodrine es efectivo para reducir la recurrencia de síncope en pacientes jóvenes y sanos con síncopes frecuentes.

Se sugiere la evaluación de pacientes con síncope vasovagal en centros con experiencia y, en caso de decir utilizar midodrine, tomar los recaudos para el seguimiento de los pacientes sabiendo que se está indicando una medicación vasoactiva, que requiere el monitoreo de la presión arterial en forma ambulatoria para asegurar que se mantenga dentro de los márgenes adecuados.

Lucas Aparicio [ Servicio de Clínica Médica, Hospital Italiano de Buenos Aires. lucas.aparicio@hospitalitaliano.org.ar ]

Silvina Dell’Era [ Servicio de Kinesiologia, Sección de Rehabilitación y Cuidados Respiratorios, Hospital Italiano de Buenos Aires. silvina.dellera@ hospitalitaliano.org.ar ]

Aparicio L, Dell’Era S. El midodrine disminuye la recurrencia de síncope vasovagal en pacientes jóvenes . Evid Actual Pract Ambul. $2021 ; 24(3): e 002158$. Available from: https://dx.doi.org/10.51987/EVIDENCIA.V24I4.6975. Comentado de: Sheldon R, et a. Midodrine for the Prevention of Vasovagal Syncope : A Randomized Clinical Trial. Ann Intern Med. 2021. doi: 10.7326/M20-5415. Epub ahead of print. PMID: 34339231.

\section{Referencias}

1. Sheldon R, Faris P, Tang A, et al. Midodrine for the Prevention of Vasovagal Syncope : A Randomized Clinical Trial. Ann Intern Med. 2021;Available from: 10.7326/M20-5415.

2. Jorge JG, Raj SR, Teixeira PS, et al. Likelihood of injury due to vasovagal syncope: a systematic review and meta-analysis. Europace. $2021 ; 23(7): 1092-1101$. Available from: 10.1093/europace/euab041.

3. $\mathrm{Ng} \mathrm{J}$, Sheldon RS, Ritchie D, et al. Reduced quality of life and greater psychological distress in vasovagal syncope patients compared to healthy individuals. Pacing Clin Electrophysiol. 2019;42(2):180-188. Available from: 10.1111/pace.13559.

4. Mosqueda-Garcia R, Furlan R, Tank J, et al. The elusive pathophysiology of neurally mediated syncope. Circulation. 2000;102(23):2898-906. Available from: 10.1161/01.cir.102.23.2898.

5. El-Sayed H, Hainsworth R. Salt supplement increases plasma volume and orthostatic tolerance in patients with unexplained syncope. Heart. 1996;75:134-174. Available from: 10.1136/hrt.75.2.134;http://dx.doi.org/10.1136/hrt.75.2.134.

6. Dijk NV, Quartieri F, Blanc JJ, et al. Effectiveness of physical counterpressure maneuvers in preventing vasovagal syncope: the Physical Counterpressure Manoeuvres Trial (PC-Trial). J Am Coll Cardiol. 2006;48(8):1652-1659. Available from: 10.1016/j.jacc.2006.06.059. 